Lei Li

Ronald Larson

\title{
Excluded volume effects on the birefringence and stress of dilute polymer solutions in extensional flow
}

Received: 7 December 1999

Accepted: 23 May 2000

\begin{abstract}
An algorithm is derived for calculating flow-induced birefringence using a bead-spring model with and without excluded volume effects. The simulation results for the bead-spring model compare well with experimental results for stress and birefringence in extensional flows of dilute solutions of polystyrene molecular weight 2 million in a filamentstretching device in both "theta"
\end{abstract}

and "good" solvents (Orr and Sridhar 1999; Sridhar et al. 2000). In a "good" solvent, both stress and birefringence rise much more rapidly with strain than in a "theta" solvent, making extensional rheology a very sensitive indicator of solvent quality.

Key words Birefringence Bead-spring model $\cdot$ Extensional flow $\cdot$ Polystyrene

Ann Arbor, MI 48109, USA

e-mail: rlarson@engin.umich.edu

\section{Introduction}

For many years, the deformation of polymer molecules in flow fields has been monitored by measurements not only of stress, but also of birefringence (Peterlin 1961, 1963; Fuller and Leal 1980; Bossart and Öttinger 1995; Doyle et al. 1998b; Mackey et al. 1999). Since neither stress nor birefringence provides a truly molecular picture of polymer deformation under flow, molecular modeling has been used to supplement experimental data in order to round out our molecular understanding. In the past, molecular modeling has been limited to simple 2-bead dumbbell models, or approximate treatments of more realistic multi-bead bead-spring or beadrod models. But in recent years computer power has developed to the point that accurate calculations using multi-bead models have become possible through Brownian dynamics simulations (Liu 1989; Hinch 1994; Fetsko and Cummings 1995; Grassia and Hinch 1996; Doyle et al. 1997; Andrews et al. 1998; Hernández Cifre and García de la Torre 1999; Wiest 1999a, b; Li et al. 2000a).

In this study, we will develop an algorithm to allow calculation of flow birefringence from Brownian dynamics simulations of bead-spring model, and we will compare calculations of both stress and birefringence from the bead-spring model with experimental measurements for dilute polymer solutions (Sridhar et al. 2000). The algorithm will be based on our earlier method that included polymer elasticity, frictional drag, and Brownian motion (Li et al. 2000), but here we will also consider excluded volume forces, which are needed to account for solvent quality effects that have been recently observed in extensional flow experiments with dilute "Boger fluids" (Solomon and Muller 1996; Sridhar et al. 2000). Excluded volume effects have already been incorporated into the beadspring model in steady-state flows by Andrews et al. (1998) and Hernández Cifre and García de la Torre (1999); we will use similar methods, but apply them to transient extensional flows.

\section{Materials and methods}

Modeling

For each link, or "Kuhn" segment of a polymer chain, the polarizability tensor can be expressed as (Fuller 1995):

$\underset{\sim}{\alpha}=\alpha_{2} \underset{\approx}{I}+\left(\alpha_{1}-\alpha_{2}\right)\left(\underset{\sim}{u_{i}} u_{i}\right)$ 
where $\alpha_{1}$ and $\alpha_{2}$ are the polarizabilities along and perpendicular to the segment, respectively; $u_{i}$ is a unit vector pointing in the direction of the segment, $u_{i}=\left(u_{i, 1} \tilde{u_{i, 2}} u_{i, 3}\right)$. The first and second part of the Eq. (1) are the isotropic and anisotropic polarizabilities, respectively.

Since each spring has $N_{k, s}$ Kuhn steps (see Fig. 1 for spring coordinates $(\mathbf{x}, \mathbf{y}, \mathbf{z}))$, and the whole molecular chain has $N_{\mathrm{s}}$ springs (see Fig. 2 for the global coordinates $(\mathbf{1}, \mathbf{2}, \mathbf{3})$ ), the polarizability tensor for the whole molecular chain is

$\underset{\approx}{\alpha}=\alpha_{2} \sum_{i=1}^{N_{s}} \sum_{j=1}^{N_{k, s}} \underset{\approx}{I}+\left(\alpha_{1}-\alpha_{2}\right) \sum_{i=1}^{N_{s}} \sum_{j=1}^{N_{k, s}}\left(\underset{\sim}{u_{j} u_{j}}\right)_{i}$

Here $i$ represents in the $i$-th spring, and $j$ represents the $j$-th link in the $i$-th spring.

The relationship between the polarizability and the index of refraction can be expressed by the Lorentz-Lorenz formula, and it can be linearized as (Larson, 1988)

$\underset{\approx}{\approx}=\frac{4 \pi}{3} \frac{\left(n_{0}^{2}+2\right)^{2}}{6 n_{0}} \underset{\approx}{\alpha}$

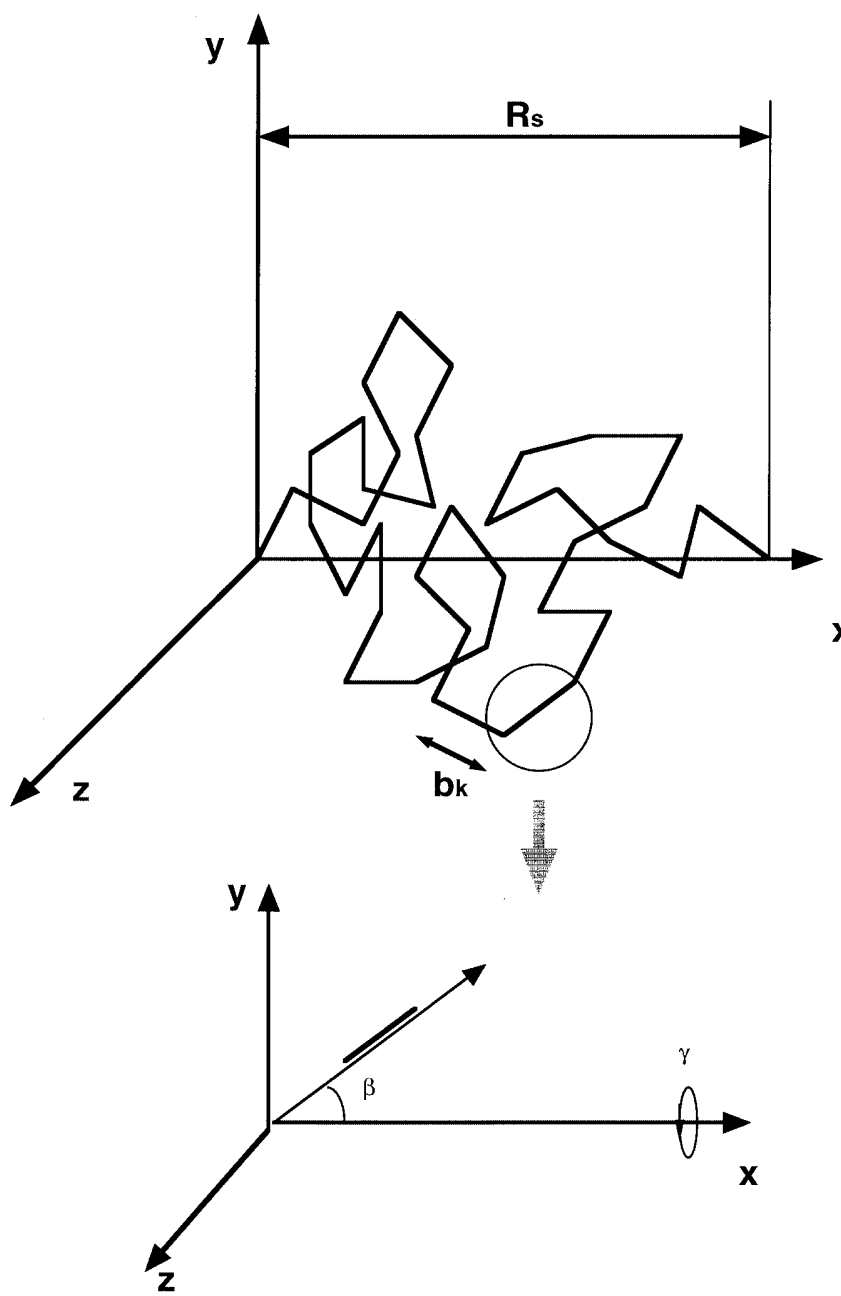

Fig. 1 Bead-rod model for a sub-chain of a polymer molecule, to be represented by a single spring in a bead-spring chain. The spring and individual rod are here shown in the "spring" frame, when the spring end-to-end vector is parallel to the $\mathrm{x}$ axis

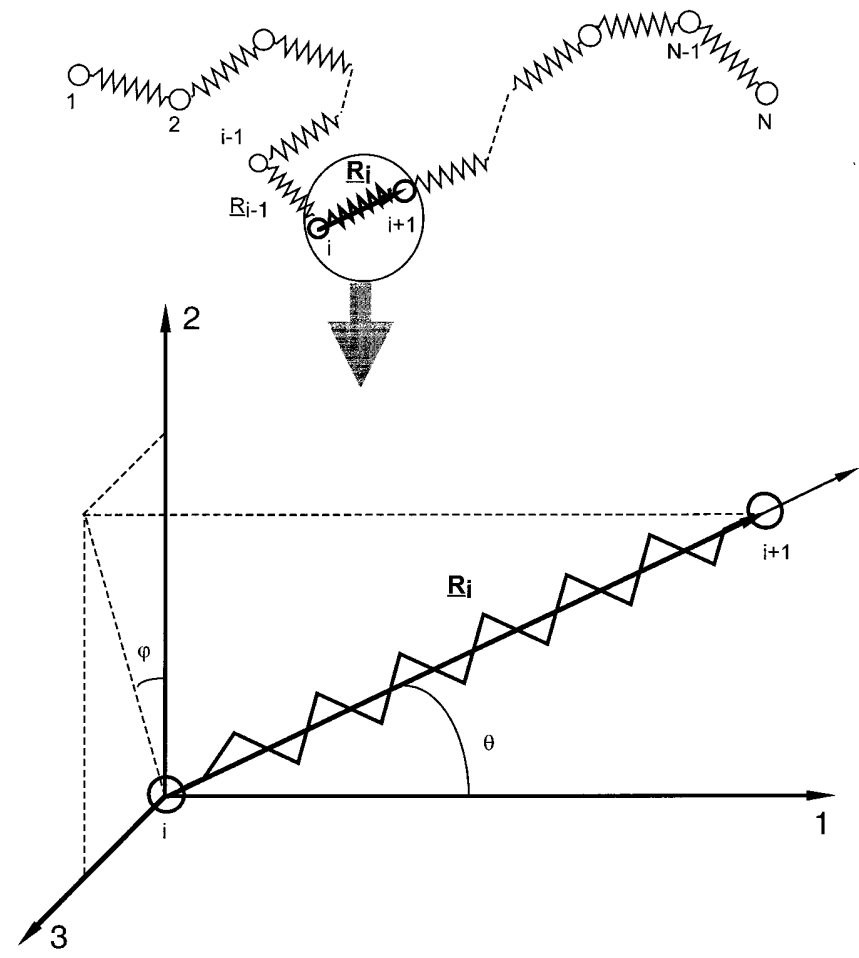

Fig. 2 The bead-spring chain and "spring" coordinates of spring $i$ in the laboratory frame

where $n_{0}$ is the refractive index of the solution. Hence, dropping the isotropic piece, the anisotropic refractive index tensor is

$\underset{\approx}{n}=\frac{4 \pi}{3} \frac{\left(n_{0}^{2}+2\right)^{2}}{6 n_{0}}\left(\alpha_{1}-\alpha_{2}\right) N_{k, s} \sum_{i=1}^{N_{s}} \sum_{j=1}^{N_{k, s}} \frac{\left(\begin{array}{c}\left.u_{j} u_{j}\right)_{i} \\ \sim\end{array}\right.}{N_{k, s}}$

The relationship between the refractive index tensor and the end-to-end distance of a spring

In the bead-spring model, we assume that each spring represents a freely jointed sub-chain containing $N_{k, s}$ Kuhn steps, and that each of these $N_{k, s}$ links in the sub-chain are thermally equilibrated subject to the constraint that the two ends of the sub-chain are held fixed at the positions of the two beads attached to the corresponding spring. The assumption that the links of the sub-chain are orientationally equilibrated subject to the constraints on the ends is the same assumption required to obtain the famous inverse Langevin spring law, and is expected to be valid as long as the flow is slow compared to the relaxation time of an individual spring. The inverse Langevin law relates analytically the spring force $f^{s}$ to the end-to-end vector $R_{s}$ of the spring in the spring frame. Then, for the birefringence tensor, we need an analytic relationship between $\sum_{j=1}^{N_{k, s}} \frac{\left(u_{j} u_{j}\right)_{i}}{\sim} \underset{\sim}{\sim}$ and $R_{s, s}$.

For each Kuhn segment, the unit vector $u_{j}$ can be expressed as

$$
\begin{aligned}
& \underset{\sim}{u_{j}}=\left(\begin{array}{lll}
u_{j, 1} & u_{j, 2} & u_{j, 3}
\end{array}\right)=\left(\begin{array}{lll}
u_{j, x} & u_{j, y} & u_{j, z}
\end{array}\right) \underset{\approx}{Q_{i}} \\
& =(\cos \beta \sin \beta \sin \gamma \sin \beta \cos \gamma) \underset{\approx}{Q_{i}}
\end{aligned}
$$


where $(\mathbf{1}, \mathbf{2}, \mathbf{3})$ represents the coordinates in the laboratory frame, and $(\mathbf{x}, \mathbf{y}, \mathbf{z})$ represents the coordinate in a frame oriented with the spring, with $\beta$ the polar and $\gamma$ the azimuthal angle of the Kuhn segment with respect to the "spring frame" (see Fig. 1). Again, $i$ represents the $\mathrm{i}$-th spring and $j$ represents the $\mathrm{j}$-th rod. The rotation tensor for spring $i$ is (Bird et al. 1987)

$\underset{\approx}{\underset{\approx}{Q}}=\left[\begin{array}{ccc}C & S c & S s \\ -S & C c & C s \\ 0 & -s & c\end{array}\right]$

Here $C=\cos \theta, S=\sin \theta, c=\cos \varphi$, and $s=\sin \varphi$. Here $\theta$ is the polar angle of the spring vector with respect to the $\mathbf{1}$ axis (the uniaxial stretching axis) of the laboratory frame, and the projection of the spring onto the $(\mathbf{2}, \mathbf{3})$ plane defines the azimuthal angle $\varphi$ (See Fig. 2).

Since the two ends of the sub-chain are held fixed, the orientation-dependent potential energy $W_{s}$ of each segment is (Larson 1999)

$W_{s}=-f^{s} b_{\kappa} \cos \beta$

where $f^{s}$ is spring force. With $C_{1}$ as the normalization constant, the distribution of each segment orientation $\psi(\beta)$ is given by the Boltzmann equation

$\psi(\beta)=C_{1} \exp \left(-\frac{W_{s}}{k_{B} T}\right)$

Then the relationship of end-to-end vector length $R_{S}$ and spring force $f^{s}$ is

$\frac{R_{s}}{L_{s}}=\langle\cos \beta\rangle=L\left(\frac{f^{s} b_{k}}{k_{B} T}\right)$

where $L_{s}$ is the fully extended length of a spring, and $L(\omega)$ is the Langevin function, $L(\omega)=\operatorname{coth}(\omega)-\frac{1}{\omega}$.

The contribution to the refractive index tensor of this multi-rod chain is

$$
\begin{aligned}
& \underset{n_{i}}{\approx}=\frac{4 \pi}{3} \frac{\left(n_{0}^{2}+2\right)^{2}}{6 n_{0}} \\
& \times\left(\alpha_{1}-\alpha_{2}\right) N_{k, s}\left[\begin{array}{lll}
\sum_{j=1}^{N_{k, s}} \frac{u_{j, 1} u_{j, 1}}{N_{k, s}} & \sum_{j=1}^{N_{k, s}} \frac{u_{j, 1} u_{j, 2}}{N_{k, s}} & \sum_{j=1}^{N_{k, s}} \frac{u_{j, 1} u_{j, 3}}{N_{k, s}} \\
\sum_{j=1}^{N_{k, s}} \frac{u_{j, 2} u_{j, 1}}{N_{k, s}} & \sum_{j=1}^{N_{k, s}} \frac{u_{j, 2} u_{j, 2}}{N_{k, s}} & \sum_{j=1}^{N_{k, s}} \frac{u_{j, 2} u_{j, 3}}{N_{k, s}} \\
\sum_{j=1}^{N_{k, s}} \frac{u_{j, 3} u_{j, 1}}{N_{k, s}} & \sum_{j=1}^{N_{k, s}} \frac{u_{j, 3} u_{j, 2}}{N_{k, s}} & \sum_{j=1}^{N_{k, s}} \frac{u_{j, 3} u_{j, s}}{N_{k, s}}
\end{array}\right]_{i} \\
& =\frac{4 \pi}{3} \frac{\left(n_{0}^{2}+2\right)^{2}}{6 n_{0}}\left(\alpha_{1}-\alpha_{2}\right) \\
& \times N_{k, s} \underset{\sim}{Q_{i}^{T}}\left[\begin{array}{ccc}
\sum_{j=1}^{N_{k, s} \frac{u_{j, x} u_{j, x}}{N_{k, s}}} & 0 & 0 \\
0 & \sum_{j=1}^{N_{k, s}} \frac{u_{j, y} u_{j, y}}{N_{k, s}} & 0 \\
0 & 0 & \sum_{j=1}^{N_{k, s}} \frac{u_{j, z} u_{j, z}}{N_{k, s}}
\end{array}\right]_{i}^{\underset{\approx}{Q_{i}}} \\
& =\frac{4 \pi}{3} \frac{\left(n_{0}^{2}+2\right)^{2}}{6 n_{0}}\left(\alpha_{1}-\alpha_{2}\right) \\
& \times N_{k, s} Q_{i}^{T}\left[\begin{array}{ccc}
\left\langle u_{j, x} u_{j, x}\right\rangle & 0 & 0 \\
0 & \left\langle u_{j, y} u_{j, y}\right\rangle & 0 \\
0 & 0 & \left\langle u_{j, z} u_{j, z}\right\rangle
\end{array}\right]_{i} \underset{\approx}{Q_{i}}
\end{aligned}
$$

$$
\begin{aligned}
& =\frac{4 \pi}{3} \frac{\left(n_{0}^{2}+2\right)^{2}}{6 n_{0}}\left(\alpha_{1}-\alpha_{2}\right) \\
& \quad \times N_{k, s} \underset{\approx}{Q_{i}^{T}}\left[\begin{array}{ccc}
f_{x x} & 0 & 0 \\
0 & f_{y y} & 0 \\
0 & 0 & f_{z z}
\end{array}\right]_{i}^{\underset{\approx}{Q_{i}}}
\end{aligned}
$$

The $f$ 's are defined by Eq. (10), where all off-diagonal terms are zero in the spring frame, by symmetry. Also, we can see that $f_{x x}+f_{y y}+f_{z z}=1$. Hence

$$
\left\{\begin{array}{l}
f_{x x}=\left\langle\cos ^{2} \beta\right\rangle=1-2 \frac{L(\omega)}{\omega} \\
f_{y y}=f_{z z}=\frac{1-f_{x x}}{2}=\frac{L(\omega)}{\omega} \\
\omega=\frac{f^{s} b_{k}}{k_{B} T}
\end{array}\right.
$$

where $L(\omega)=\frac{R_{s}}{L_{s}}$ and $\omega$ is given by the inverse Langevin function, $\omega=L^{-1}\left(\frac{R_{s}}{L_{s}}\right)$.

Since the Langevin and the inverse Langevin functions are used in Eq. (11), there is no analytic relationship between $\sum_{j=1}^{N_{k, s}} \frac{\left(u_{j} u_{j}\right)_{i}}{N_{k, s}}$

and $R_{s}$. We can, however, use an approximation for the inverse Langevin function, such as the Warner spring law, or the more accurate Cohen (1991) Padé approximation, or we can perform a Monte Carlo simulation to obtain the relationship.

For the Cohen approximation, we have

$L^{-1}\left(\frac{R_{s}}{L_{s}}=\lambda_{i}\right)=\lambda_{i} \frac{3-\lambda_{i}^{2}}{1-\lambda_{i}^{2}}+O\left(\lambda_{i}^{6}\right)$

where $\lambda_{i}^{2}=\frac{\left(R_{s}^{2}\right)_{i}}{L_{s}^{2}}$ is the ratio of the square of the end-to-end distance of the multi-rod sub-chain representing the $i$-th spring $\left(R_{s}^{2}\right)_{i}$ to the square of the length of a fully extended multi-rod sub-chain $\left(L_{s}^{2}\right)$.

The Cohen approximation does not give the correct value of $f_{x x}$ and $f_{y y}$ for small stretch $R_{s} / L_{s}$. Therefore, we performed Monte Carlo simulation for both 10-rod and 50-rod freely jointed chains, and obtained the relationship between $\sum_{j=1}^{N_{k, s}}\left(u_{j} u_{j}\right)_{i} / N_{k, s}$ and $R_{s}$ in spring coordinates $(\mathbf{x}, \mathbf{y}, \mathbf{z})$; see Fig. 2. As can $\tilde{\text { be }} \tilde{\tilde{s}}$ seen in Fig. 3 , the following third-order polynomials give the correct asymptotic results in the limits of both large and small birefringence and provide an excellent fit to the simulation curves for both 10-rod and 50-rod chains:

$$
\begin{aligned}
& f_{x x}=\frac{1}{3}+\frac{2}{5}\left(\lambda_{i}^{2}\right)+\frac{2}{15}\left(\lambda_{i}^{2}\right)^{2}+\frac{2}{15}\left(\lambda_{i}^{2}\right)^{3} \\
& f_{y y}=\frac{1}{3}-\frac{1}{5}\left(\lambda_{i}^{2}\right)-\frac{1}{15}\left(\lambda_{i}^{2}\right)^{2}-\frac{1}{15}\left(\lambda_{i}^{2}\right)^{3} \\
& f_{x x}-f_{y y}=\frac{1}{5}\left(\left(\lambda_{i}^{2}\right)^{3}+\left(\lambda_{i}^{2}\right)^{2}+3\left(\lambda_{i}^{2}\right)\right)
\end{aligned}
$$

Since each multi-rod chain is a spring in the bead-spring model, its end-to-end vector is not necessarily aligned along the extension direction (here the 1 direction); see Fig. 2. Thus, to calculate the contribution to the overall refractive index tensor from each spring, we must rotate the tensor as shown by Eq. (10), where the rotation tensor $Q$ is defined by Eq. (6) (Bird et al. 1987). Then the refractive index tẽ̃nsor becomes

$\underset{n_{i}}{\approx}=\frac{4 \pi}{3} \frac{\left(n_{0}^{2}+2\right)^{2}}{6 n_{0}}\left(\alpha_{1}-\alpha_{2}\right) N_{k, s}\left[\begin{array}{ccc}T_{11} & T_{12} & T_{13} \\ T_{21} & T_{22} & T_{23} \\ T_{31} & T_{32} & T_{33}\end{array}\right]_{i}$ 


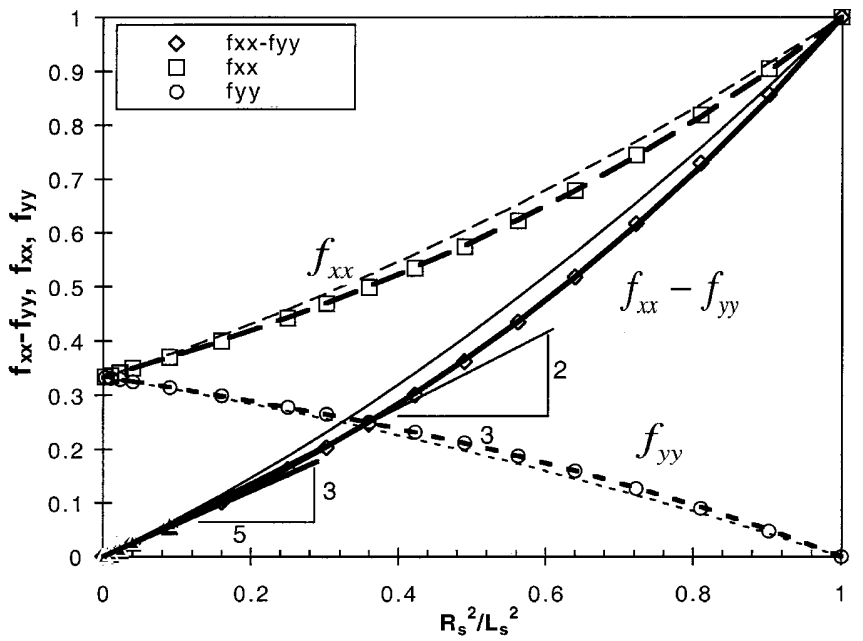

Fig. 3 The relationship of normalized components of the refractive index tensor to the overall stretch ratio squared $R_{s}^{2} / \mathrm{L}^{2}$. The squares, circles and diamonds are Monte Carlo simulation results respectively for $f_{x x}, f_{y y}$, and $f_{x x}-f_{y y}$ vs stretch ratio squared $R_{s}^{2} / \mathrm{L}^{2}$. The thick solid and dashed lines are best fits of third-order polynomials (Eq. 13) for the simulation results for the 50-rod chain. The thin lines are calculations from Cohen approximation, Eqs. (11) and (12)

where

$$
\begin{aligned}
& T_{11, i}=\left\langle C^{2} f_{x x}+S^{2} f_{y y}\right\rangle_{i} \\
& T_{12, i}=T_{21, i}=\left\langle\operatorname{CSc}\left(f_{x x}-f_{y y}\right)\right\rangle_{i} \\
& T_{13, i}=T_{31, i}=\left\langle\operatorname{CSs}\left(f_{x x}-f_{y y}\right)\right\rangle_{i} \\
& T_{22, i}=\left\langle S^{2} c^{2} f_{x x}+C^{2} c^{2} f_{y y}+s^{2} f_{y y}\right\rangle_{i} \\
& T_{23, i}=T_{32, i}=\left\langle c s\left(f_{x x}+f_{y y}-f_{y y}\right)\right\rangle_{i} \\
& T_{33, i}=T_{33, i}=\left\langle S^{2} s^{2} f_{x x}+C^{2} s^{2} f_{y y}+c^{2} f_{y y}\right\rangle_{i}
\end{aligned}
$$

Because the spring orientation in a uniaxial flow is symmetric about $\theta=0$ and isotropic in the angle $\varphi$, we have $\left\langle s^{2}\right\rangle=\left\langle c^{2}\right\rangle=\frac{1}{2}$, and $\langle\mathrm{SC}\rangle=\langle\mathrm{sc}\rangle=0$. Then the birefringence, which is the difference of two eigenvalues of the refractive index tensor, is

$$
\begin{aligned}
\Delta n_{i}= & \Delta n_{i, x x}-\Delta n_{i, y y} \\
= & \frac{4 \pi}{3} \frac{\left(n_{0}^{2}+2\right)^{2}}{6 n_{0}}\left(\alpha_{1}-\alpha_{2}\right) N_{k, s} \\
& \times\left(\left\langle C^{2} f_{x x}+S^{2} f_{y y}\right\rangle_{i}-\left\langle S^{2} c^{2} f_{x x}+C^{2} c^{2} f_{y y}+s^{2} f_{y y}\right\rangle_{i}\right) \\
= & \frac{4 \pi}{3} \frac{\left(n_{0}^{2}+2\right)^{2}}{6 n_{0}}\left(\alpha_{1}-\alpha_{2}\right) N_{k, s}\left\langle\left(\frac{3}{2} C^{2}-\frac{1}{2}\right)\left(f_{x x}-f_{y y}\right)\right\rangle_{i}
\end{aligned}
$$

Thus, the contribution of the whole polymer (all springs) to the birefringence is

$$
\begin{aligned}
\Delta n= & \frac{4 \pi}{3} \frac{\left(n_{0}^{2}+2\right)^{2}}{6 n_{0}}\left(\alpha_{1}-\alpha_{2}\right) v N_{k, s} \\
& \times \sum_{i=1}^{N_{s}}\left\langle\left(\frac{3}{2} C^{2}-\frac{1}{2}\right)\left(f_{x x}-f_{y y}\right)\right\rangle_{i}
\end{aligned}
$$

where $v=\frac{N_{A} c_{\mathrm{m}} \rho}{M}$ is the number of molecules per unit volume, $c_{\mathrm{m}}$ is the polymer mass fraction, and $\rho$ is the solution density.

\section{Brownian dynamics simulations of bead-spring model}

In our Brownian dynamics simulations, hydrodynamic interactions are considered only through our choice of bead drag coefficients and we use the accurate Cohen (1991) Padé approximation to approximate the inverse Langevin function used in the force law for the freely jointed chain. Hence, we solve ( $\mathrm{Li}$ et al. 2000)

$$
\begin{aligned}
\underset{\sim i}{\mathrm{~d} r}=\underset{\approx}{\kappa} \cdot \underset{\sim i}{r}+\frac{k_{B} T}{b_{K} \zeta} \lambda_{i} \frac{3-\lambda_{i}^{2}}{1-\lambda_{i}^{2}} \underset{\sim i}{\stackrel{R}{R_{i}}} \\
-\frac{k_{B} T}{b_{K} \zeta} \lambda_{i-1} \frac{3-\lambda_{i-1}^{2}}{1-\lambda_{i-1}^{2}} \frac{\underset{\sim i-1}{R}-\left(\frac{6 k_{B} T}{\zeta \Delta t}\right)^{\frac{1}{2}} \underset{\sim i}{R_{i-1}}}{n}
\end{aligned}
$$

Here $\kappa=\nabla v^{T}$ is the transpose of the velocity gradient tensor, $\lambda_{i}$ is the extension ratio

$\lambda_{i}=\frac{R_{i}}{L_{i}}=\frac{R_{i}}{N_{K, s} b_{K}}$

and $R_{i}$ is the extension of spring $i$ in the laboratory frame (see Fig. 2):

$R_{i} \equiv|\underset{\sim i+1}{r}-\underset{\sim i}{r}| ; \quad \underset{\sim i}{R} \equiv \underset{\sim i+1}{r}-\underset{\sim i}{r}$

$b_{K}$ being the Kuhn step length of the freely jointed chain, and $N_{K, s}$ the number of Kuhn steps per spring. Thus $L_{\mathrm{s}}=N_{K, s} \mathrm{~b}_{K}$ is the fully extended length of a spring. For polystyrene, $b_{K}$ is given by

$b_{K}=\frac{C_{\infty}}{n} l=1.803 \times 10^{-3} \mu m$

where $C_{\infty}$ is the molecular characteristic ratio, which is $C_{\infty}=9.6$ for polystyrene; $n$ is the number of back-bone bonds in one polymer molecule, $n=\frac{M}{52}$ for polystyrene, and $M$ is the polymer molecular weight.

According to polymer theory (Bird et al. 1987), the stress tensor can be expressed as

$\underset{\approx}{\sigma}=\underset{\approx}{\sigma^{p}}+\eta_{s}\left(\underset{\approx}{\kappa}+\underset{\approx}{\kappa^{T}}\right)$

$\underset{\approx}{\sigma^{p}}=v \sum_{i=1}^{N-1}\left\langle\underset{\sim i}{F^{s p}} \underset{\sim i}{R}\right\rangle-v(N-1) k_{B} T \underset{\approx}{I}$

where $I$ is the unit tensor, $v=\frac{N_{A} c_{m} \rho}{M}$ is the number of polymer molecules per unit volume of solution, $\rho$ is the density of the solution, $\rho=1.04 \mathrm{~g} / \mathrm{cm}^{3}$ for polystyrene, $\eta_{s}=30$ Pas is the solvent viscosity for an approximate "theta" solvent studied by Orr and Sridhar (1999), and $\eta_{s}=87.5$ Pas for a "good" solvent for polystyrene studied by Sridhar et al. (2000); $\sigma_{\sim}^{p}$ is the polymer contribution to the stress tensor, and $\eta_{s}(\underset{\approx}{\kappa+\underset{\approx}{\kappa}})$ is the solvent contribution.

\section{Solvent quality}

"Theta" solvent. As described in detail in our previous paper ( $\mathrm{Li}$ et al. 2000), we use molecular theories, namely the Zimm theory for a polymer in a theta solvent, and also the molecular characteristics of polystyrene, to determine a priori parameters for our simulations. The results of those simulations were compared to extensional flow data for high molecular-weight polystyrene in a dilute solution in a solvent consisting of $25 \mathrm{wt} \%$ low molecular weight $(M=50,000)$ polystyrene (PS) in dioctyl phthalate (DOP) at $21^{\circ} \mathrm{C}$.

Since DOP is a theta solvent for polystyrene at this temperature, we assumed earlier ( $\mathrm{Li}$ et al. 2000) and in what follows that the 
PS/DOP binary solvent is also a theta solvent, and use the corresponding Zimm theory for a theta solvent to obtain the following expressions for the bead drag coefficient $\zeta^{Z}$ :

$$
\begin{aligned}
\frac{\zeta^{Z}}{k_{B} T}= & \frac{K_{\theta} M^{\frac{1}{2}} \eta_{s}}{N_{A} k_{B} T} \frac{1}{2.369} \\
& \times \frac{6 \times 52 \pi^{2}}{C_{\infty} l^{2}} \frac{N_{s}}{\left(N_{s}+1\right)^{2}}=367.5 \frac{s}{\mu \mathrm{m}^{2}}
\end{aligned}
$$

Here $K_{\theta}=8 \times 10^{-4}$ deciliters $\mathrm{g}^{-1}(\mathrm{~g} / \mathrm{mole})^{1 / 2}$ is a "universal" number for theta solvents, $N_{A}$ is Avogadro's number, and $N_{s}$ is number of springs; $N_{s}=19$ in our simulations.

Hence the longest Zimm relaxation time is

$\tau_{\text {cal }}^{Z}=\frac{K_{\theta} M^{\frac{3}{2}} \eta_{s}}{2.369 N_{A} k_{B} T}=1.11 s$

where we have use the experimental values $M=2 \times 10^{6}$ Daltons and $\eta_{\mathrm{s}}=30$ Pas.

"Good" solvent. Since the quality of a solvent for a given polymer affects the coil size of that polymer at equilibrium, it will also affect the unraveling of the polymer in an extensional flow (Larson 1988; Solomon and Muller 1996). Intrinsic viscosity experiments show that the coil size of polystyrene in "piccolastic," a low molecularweight styrene oligomer, is larger than in a mixture of low molecular-weight polystyrene (PS) with dioctylphthate (DOP), where DOP is a theta solvent for polystyrene at the experimental temperature $\left(21^{\circ} \mathrm{C}\right)$. In our simulations, we treat the PS/DOP mixture as a theta solvent for high molecular-weight polystyrene, while we consider piccolastic a "good" or at least "better-thantheta" solvent, for which excluded-volume (EV) effects must be incorporated. We include EV effects by invoking a truncated Lennard-Jones potential between each pair of beads on the chain; i.e., (Rzehak et al. 1999)

$$
\begin{aligned}
W^{\mathrm{L}-\mathrm{J}}(\underset{\sim}{r})= & 4 k_{B} T \varepsilon\left((\underset{\sim}{|\underset{\sim}{r}|})^{12}-\left(\frac{\sigma}{|\underset{\sim}{r}|}\right)^{6}+\frac{1}{4}\right) \\
& \times \Theta\left(|\underset{\sim}{r}|-R_{\mathrm{LJ}}\right)
\end{aligned}
$$

The cut-off radius $R_{\mathrm{LJ}}$ is chosen at the minimum of the LennardJones potential, $R_{\mathrm{LJ}}=2^{1 / 6} \sigma ; \Theta(x)$ is the Heaviside function. We set the dimensionless energy parameter $\varepsilon$ to the value $\varepsilon=1.0$ and we set $\sigma$, the Lennard-Jones radius, to $\sigma=4 a$, where $a$ is the effective bead radius, related to the bead drag coefficient $\zeta$ by $\zeta=6 \pi \eta_{\mathrm{s}} \mathrm{a}$.

From the experimental data (see Table 1), the relaxation time for piccolastic "good" solvent is about ten times larger than that in PS/DOP "theta" solvent, although the solvent viscosity of the former is only three times larger then that of the latter (87.5 Pas vs 30 Pas). Hence the expression for the drag coefficient formula for a "theta" solvent is evidently not valid any more in the "good" solvent case. Therefore, we use instead a "Rouse" drag coefficient, in which $\zeta$ is chosen so that the drag on a fully extended chain in the extensional flow matches the Batchelor formula for the drag on a slender cylinder (Batchelor 1970; Li et al. 2000):

$\frac{\zeta^{R}}{k_{B} T}=\eta_{s} \frac{2 \pi}{\ln \left(\frac{L}{d}\right)} \frac{L}{N}$

where $L$ is the fully extended length of the molecule and $d$ is its effective diameter, which for polystyrene we take to be $0.0009 \mu \mathrm{m}$. Then the bead radius $a$ can be calculated by $a=\zeta^{R} / 6 \pi \eta_{s}$. Hence, the relaxation time can be calculated as

$\tau_{c a l}^{R}=\frac{\zeta^{R}}{16 k_{B} T \beta_{s}^{2} \sin ^{2}\left(\frac{\pi}{2 N}\right)} \approx \frac{N_{K} b_{K}^{2} L \eta_{s}}{3 \pi \ln \left(\frac{L}{d}\right)}$
Table 1 Relaxation time comparison

\begin{tabular}{lrrrrr}
\hline & $\begin{array}{c}\tau^{\mathrm{R}}{ }_{\mathrm{cal}}^{\mathrm{a}} \\
(\mathrm{sec})\end{array}$ & $\begin{array}{l}\tau^{\mathrm{R}}{ }_{\text {sim }}{ }^{\mathrm{b}} \\
(\mathrm{sec})\end{array}$ & $\begin{array}{l}\tau^{\mathrm{Z}}{ }_{\text {sim }}{ }^{\mathrm{b}} \\
(\mathrm{sec})\end{array}$ & $\begin{array}{r}\tau^{\mathrm{Z}}{ }_{\text {cal }}{ }^{\mathrm{c}} \\
(\mathrm{sec})\end{array}$ & $\begin{array}{l}\tau_{\text {exp }}{ }^{\mathrm{d}} \\
(\mathrm{sec})\end{array}$ \\
\hline 2 M PS in good solv. & 9.86 & 8.13 & 2.48 & 3.24 & 8.4 \\
2 M PS in theta solv. & 3.64 & 3.44 & 1.05 & 1.11 & 0.8 \\
10 M PS in theta solv. & 80.92 & 77.78 & 12.45 & 13.37 & 9.5 \\
\hline
\end{tabular}

${ }^{\mathrm{a}} \tau_{\mathrm{R}}^{\mathrm{R}}$ are calculated from Eq. (27)

${ }^{\mathrm{b}} \tau^{\mathrm{R}}{ }_{\text {sim }}^{\mathrm{cal}}$ and $\tau^{\mathrm{Z}}{ }_{\text {sim }}$ are calculated from Eq. (28) and they are the average of 50 individual molecules

${ }^{c} \tau^{Z}$ cal are calculated from Eq. (24)

${ }^{\mathrm{d}} \tau_{\exp }$ are from experiments (Orr and Sridhar 1999; Sridhar et al. 2000)

where $\beta_{s}^{2}=\frac{3}{2 N_{K, s} b_{K}^{2}}=\frac{3 N_{K}}{2 N_{s} b_{K}^{2}}$, and $N_{K}=\frac{(0.82)^{2}}{C_{\infty}} n$ is the number of Kuhn steps in a whole molecule.

For 2 million molecular-weight polystyrene in piccolastic "good" solvent, we can calculate from Eq. (26) (for 10 beads) that $\frac{\zeta^{R}}{k_{B} T}=6670 \frac{s}{\mu m^{2}}$, which is about ten times larger than our drag coefficient for the same polymer in PS/DOP, and about three times larger than the value we would have obtained for piccolastic had we used the "Zimm" value of $\zeta^{z} / k_{B} T$.

We also calculate the relaxation of an ensemble of polymer chains using Brownian dynamics simulations, and then fit the last $9 \%$ of the end-to-end distance squared $\left\langle R^{2}\right\rangle$ to the equation

$\left\langle R^{2}\right\rangle=C \exp (-t / \tau)+\left\langle R^{2}\right\rangle_{0}$

By fitting this expression to the simulation data we can obtain another estimate of the relaxation time of the polymer chain, $\tau_{\text {sim. }}$. Estimates of the relaxation times obtained in this way are tabulated in Table 1, where $\tau^{\mathrm{R}}$ sim is obtained using the "Rouse" formula, Eq. (26), to assign the bead drag coefficient, $\tau^{Z}{ }_{\text {sim }}$ being obtained using the "Zimm" formula, Eq. (23). In all cases, the values of $\tau^{\mathrm{R}}{ }_{\text {sim }}$ and $\tau^{\mathrm{Z}}{ }_{\text {sim }}$ are in good agreement with the corresponding calculated values from Eqs. (27) and (24), respectively. In addition, note that in the "theta" solvent the "Zimm" relaxation times $\tau^{\mathcal{Z}}{ }_{\text {sim }}$ and $\tau_{\text {cal }}^{Z}$ are in reasonable agreement with the experimental relaxation time $\tau_{\exp }$, while in the good solvent the "Rouse" relaxation times $\tau^{\mathrm{R}}$ sim and $\tau^{\mathrm{R}}$ cal are in much better agreement with $\tau_{\exp }$ than are the Zimm relaxation times. Thus, for the "theta" solvent PS/DOP, we will use $\zeta^{Z}$ from Eq. (23) for the bead drag coefficient in our simulations, while for the "good" solvent piccolastic, we will use $\zeta^{\mathrm{R}}$ from Eq. (26). In all simulations, we impose an extension rate $\dot{\varepsilon}$ chosen to match the experimental values. The Deborah numbers $D e=\dot{\varepsilon} \tau_{\text {sim }}$ in our simulations therefore match the experimentally assigned De values to the extent that $\tau_{\text {sim }}\left(=\tau^{\mathrm{R}}\right.$ sim for a "good" solvent, $\tau_{\text {sim }}^{\mathrm{Z}}$ for a "theta" solvent) matches the experimental relaxation time $\tau_{\text {exp }}$. Finally, we note that each experimental relaxation time was obtained by Sridhar and coworkers as the largest relaxation time from a fit of the Zimm spacing of relaxation times to the experimental dynamic oscillating shear data (a fit of the Rouse spacing gives nearly the same result). This experimental relaxation time should be very nearly the longest relaxation time of the solution, and hence should correspond well with the value $\tau_{\text {sim }}$ or $\tau_{\mathrm{cal}}$ from bead-spring model. We also obtain from the simulations the mean end-to-end distance squared in the rest state, $\left\langle R^{2}\right\rangle_{\mathrm{o}}$, by averaging $R^{2}$ in the absence of flow over a period of time, which is more than 1000 times the relaxation time.

For the birefringence calculations, we use Eqs. (17) and (13), where $n_{\mathrm{o}}=1.6$ is the refractive index of the solution (Bradrup and Immergut 1989); $\left(\alpha_{1}-\alpha_{2}\right)$ is the polarizability difference, $\left(\alpha_{1}-\right.$ $\alpha_{2)}=-145 \times 10^{-25} \mathrm{~cm}^{3}$ for polystyrene (Bradrup and Immergut 1989). From these calculations, we obtain a value for the stress- 
optic coefficient of $C_{\Delta n}=\frac{2 \pi}{45 k_{B} T} \frac{\left(n_{0}^{2}+2\right)^{2}}{n_{0}}\left(\alpha_{1}-\alpha_{2}\right)=-6.4 \times 10^{-9} \mathrm{~Pa}^{-1}$, which differs somewhat from the value $C_{\Delta \mathrm{n}}=5 \times 10^{-9} \mathrm{~Pa}^{-1}$ used by Sridhar and coworkers. For consistency, we will use the value $-5.0 \times 10^{-9} \mathrm{~Pa}^{-1}$ in our comparisons of experiments with simulations (i.e., in Figs. 7 and 8).

\section{Results and discussion}

Comparison with bead-rod model

To check our algorithm for calculating the birefringence for the bead-spring model, we first performed a simulation for a 20 -spring chain in uniaxial extensional flow with no excluded volume at a Deborah number of $\mathrm{De}=10.65$, and compared the results with those of Doyle and Shaqfeh's (1998) for a 200-rod bead-rod chain at the same De, as shown in Fig. 4. For this comparison, we chose $N_{\mathrm{K}, \mathrm{s}}=10$ in the bead-spring chain, since each spring of a 20 -spring bead-spring chain corresponds to 10 rods in a 200-rod bead-rod chain. The results of the beadspring simulations agree almost perfectly with those of the bead-rod simulations. This confirms the assumption of local equilibrium within each sub-chain corresponding to an individual spring in the bead-spring chain.

Comparison with the experimental data

\section{Relaxation time comparison}

As described earlier, excluded volume was incorporated into our simulations, and we first carry out the

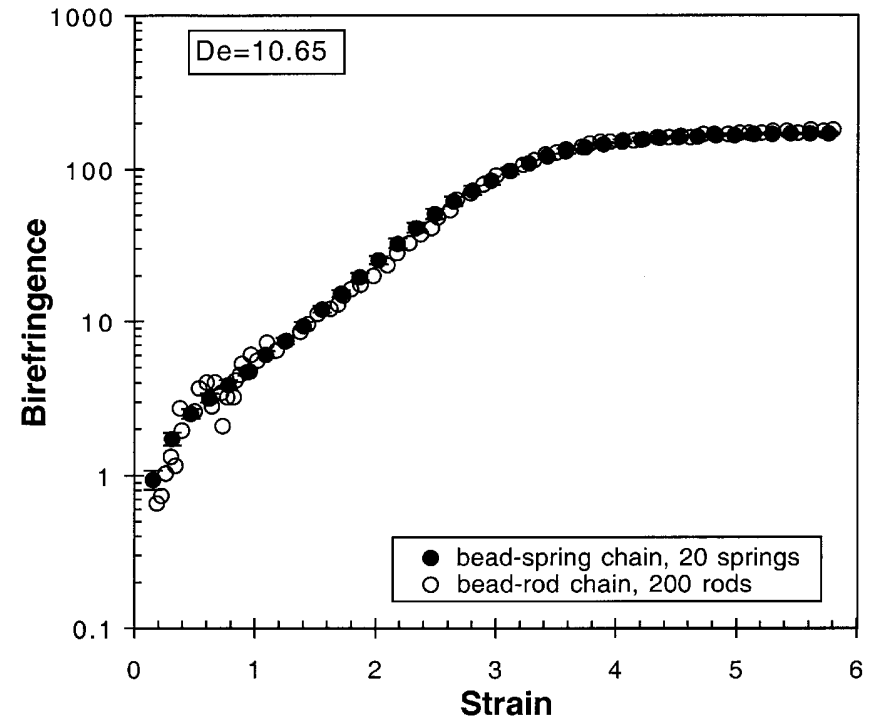

Fig. 4 Comparison of predictions of the bead-spring and bead-rod models. The open circles are the simulation results of Doyle et al. (1998) for a 200-rod bead-rod chain, and the solid circles are simulation results for a 20 -spring bead-spring model; both simulations are for extensional flow at $\mathrm{De}=10.65$ relaxation calculations for 2 million molecular-weight polystyrene. In Fig. 5, we can find that when we include the truncated LJ potential (Eq. 25), the relaxation becomes faster than without the LJ potential, and the equilibrium end-to-end length squared increases. From our calculations of $\left\langle R_{g}^{2}\right\rangle_{0}^{1 / 2}$ ( $\mathrm{Li}$ and Larson 2000), we find that the coil size increases about $60 \%$, which is from $0.0419 \mu \mathrm{m}$ to $0.0689 \mu \mathrm{m}$ when excluded volume is included. These values of the radius of gyration correspond closely to the values of $\left\langle R_{g}^{2}\right\rangle^{1 / 2}$ measured for polystyrene, respectively, in a known theta solvent, dioctyl phthalate and in a known good solvent, toluene (Solomon and Muller 1996). The relaxation time calcu-
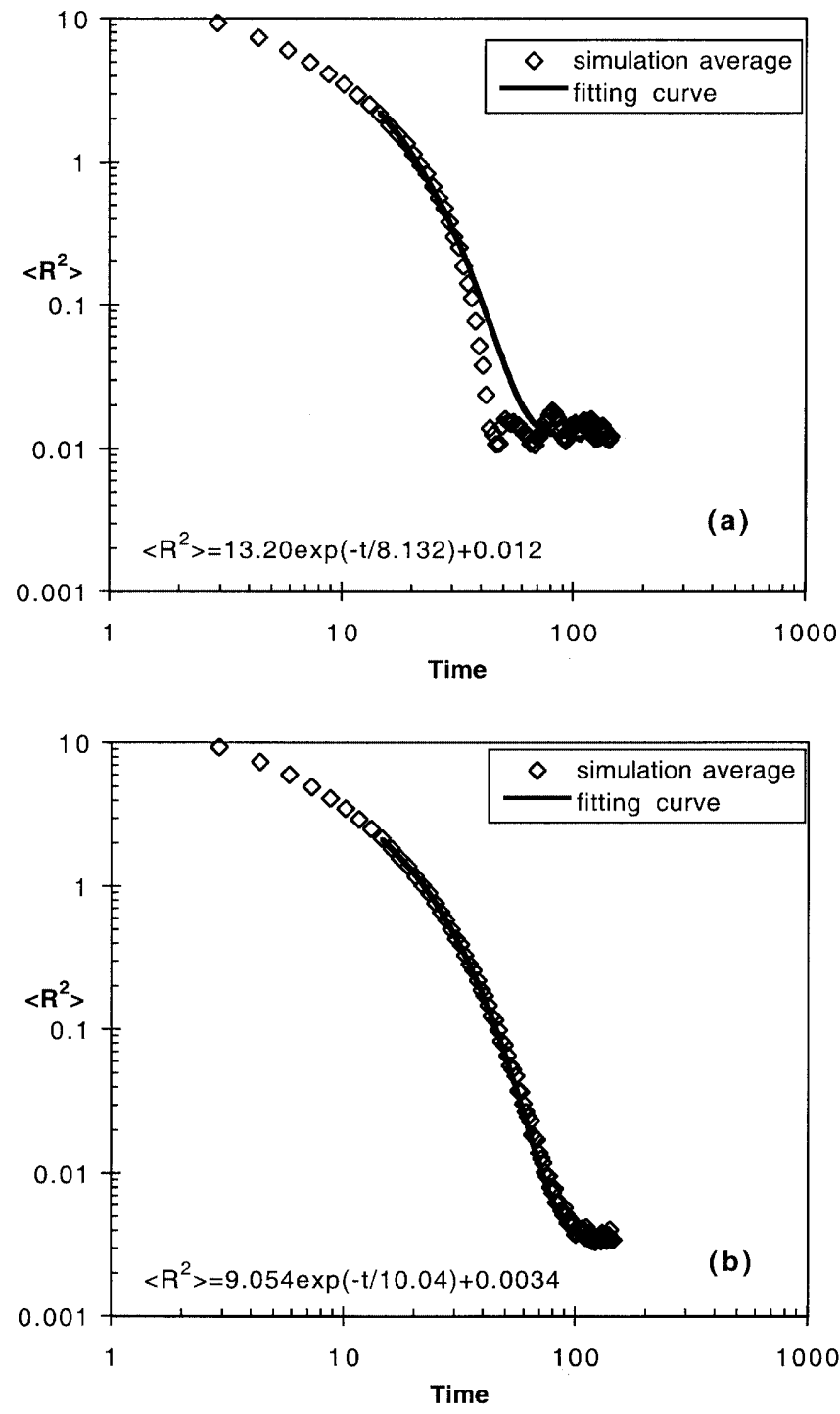

Fig. 5a, b Simulation results for the relaxation of the square end-toend vector length $R^{2}$ averaged over 50 individual molecules (symbols), and fits of an exponential decay to obtain the relaxation time $\tau$ (lines): a with excluded volume, using $\sigma=4 a=0.6632 \mu \mathrm{m}$; b without excluded volume 
lated with excluded volume is $8.13 \mathrm{~s}$, which is about the same as Sridhar's result, $8.4 \mathrm{~s}$, from dynamic oscillatory experiments (Sridhar et. al. 2000). Notice in Fig. 5a that, for a good solvent, the relaxation of $\left\langle\mathrm{R}^{2}\right\rangle$ has a steep tail that is not particularly well fit by an exponential relaxation.

\section{Comparison of stress and birefringence predictions to experimental measurements}

We performed Brownian dynamics simulations for solutions of polystyrene of molecular weight 2 million studied experimentally by Sridhar et al. (2000), and compared the predictions of both birefringence growth and stress growth with the experimental results. From Fig. 6 we can see that the theories for different solvent quality predict quite different results. The Trouton ratio begins to rise rapidly at about 2.0 Hencky strain units for a "good" solvent (piccolastic) while the rapid rise only begins at about 3.0 Hencky strain units for "theta" solvent (PS/DOP) for the same 2.0 million molecular weight polystyrene polymer. The predicted stress shows excellent agreement with the experimental results for both the "theta" solvent and "good" solvent conditions.

In Fig. 7 we compare the stress and birefringence predicted for good-solvent conditions with measure-

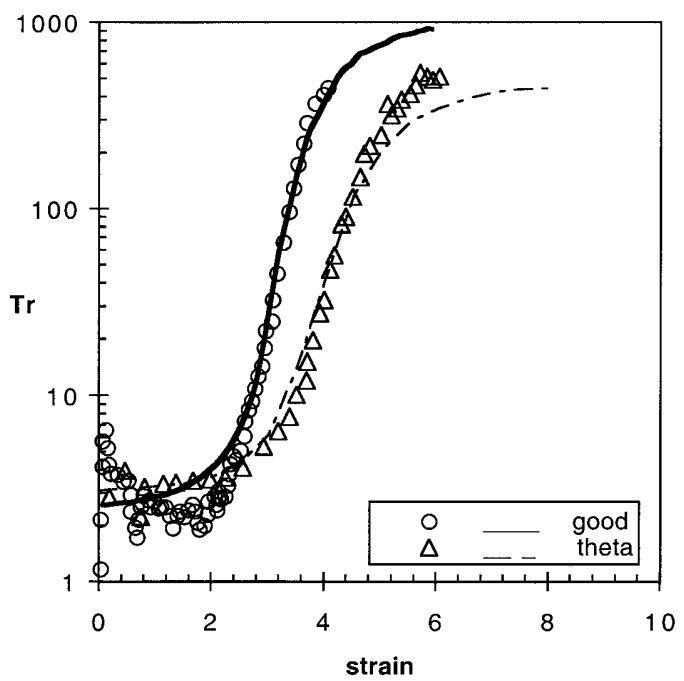

Fig. 6 Comparison of the growth of the Trouton ratio after start-up of uniaxial extensional flow for polystyrene of molecular weight 2 million at a concentration of $0.05 \%$ in "good" solvent and $0.0747 \%$ in the "theta" solvent. The thick solid line and circles are for polystyrene in a "good" solvent (piccolastic) at an extensional rate of $4.91 / \mathrm{s}$; The thin dashed line and triangles are for polystyrene in a "theta" solvent (PS/DOP) at an extension rate of $5.051 / \mathrm{s}$. The lines are simulation results averaged in this and subsequent figures over an ensemble of 100 molecules and the symbols are experimental results. For the simulations in a "good" solvent, in this and subsequent figures, we used 10 beads and parameter values $\varepsilon=1.0, \sigma=4 a=0.6632 \mu \mathrm{m}$ in Eq. (25)
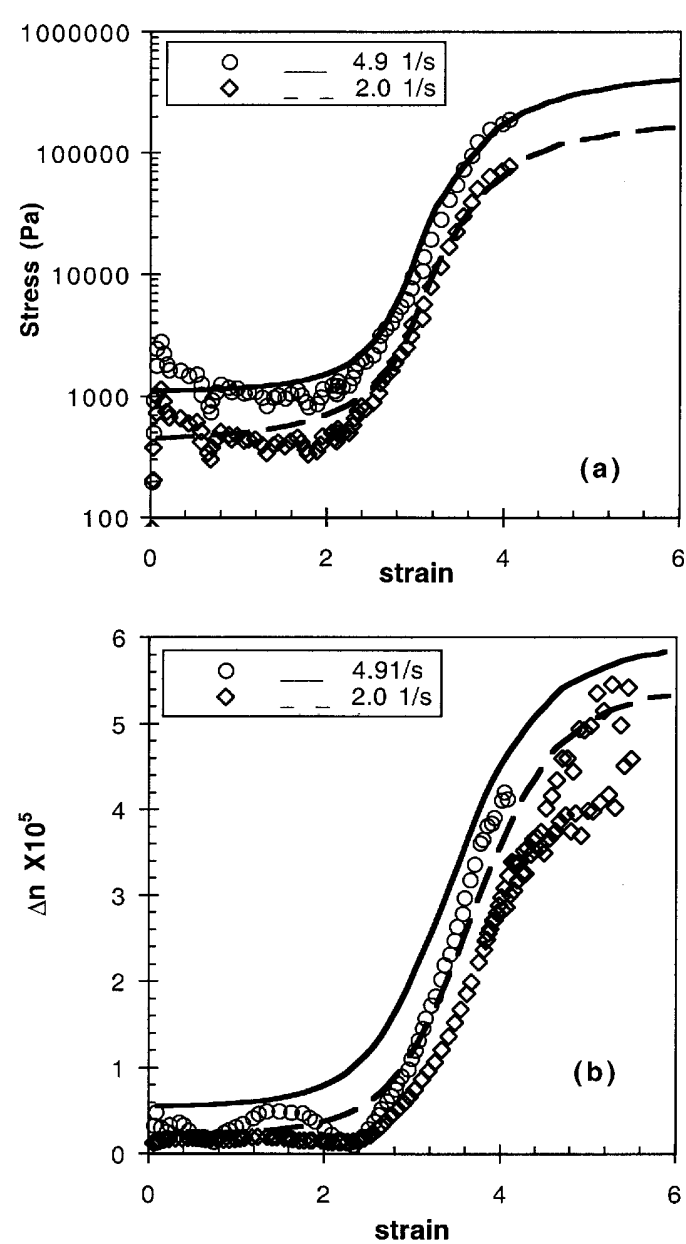

Fig. 7a, b Comparison of bead-spring simulations with experimental data for a polystyrene solution of molecular weight 2 million in a "good" solvent by Sridhar et al. (2000): a comparison of stress growth at extension rate $2.01 / \mathrm{s}$ (diamonds and dashed line) and $4.9 \mathrm{1} / \mathrm{s}$ (circles and solid line); $\mathbf{b}$ comparison of polymer contribution to birefringence growth at extension rate $2.01 / \mathrm{s}$ (diamonds and dashed line) and $4.91 / \mathrm{s}$ (circles and solid line). All symbols are experimental results and lines are simulation results

ments on 2 million molecular-weight polystyrene in piccolastic at two extension rates, $2.0 \mathrm{~s}^{-1}$ and $4.0 \mathrm{~s}^{-1}$. While the stress predictions are in good agreement with the experimental data at both extension rates (Fig. 7a), the birefringence is predicted to rise somewhat more quickly than is observed experimentally (Fig. 7b).

In Fig. 8 we show "hysteresis" curves of stress vs birefringence during start-up of extensional flow and during relaxation after cessation of flow for two different Deborah numbers. Although the previous study shows qualitative agreement with experimental measurements using the dumbbell model (Doyle et al. 1998), agreement between simulations and experiment is reasonable using a multi bead-spring model in this study, except that the simulations predict an overly 


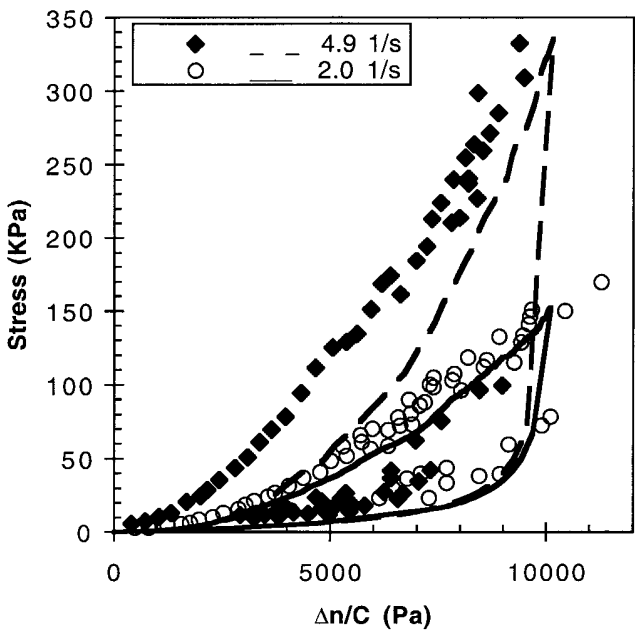

Fig. 8 Stress-optical hysteresis for two different Deborah numbers. The solid line and the circles are for $\mathrm{De}=41.7 \dot{\varepsilon}=4.96$, and the dashed line and the diamonds are for $\mathrm{De}=16.8 \dot{\varepsilon}=2.0$. The lines are simulation results and symbols are experimental results

rapid rise in birefringence and an overly rapid relaxation of stress following cessation of extension.

The much more rapid growth of stress for polystyrene in the "good" solvent relative to the "theta" solvent (Fig. 6) suggests a possible explanation for disagreements we observed earlier between simulations and experimental extensional flows of higher molecular weight polystyrene $\left(4-20 \times 10^{6}\right)$ in PS/DOP $(\mathrm{Li}$ et al. 2000). In the earlier simulations, the solvent was taken to be theta solvent, an approximation that leads to good agreement with the experimental data for polystyrene with $M=2 \times 10^{6}$. However, if the solvent quality is slightly better than theta, one would expect the swelling of the coil to become more evident as the polystyrene molecular weight increases, and for the measured growth of Trouton ratio to begin to run more and more ahead of the predicted growth as $M$ increases, which is what was observed. Because strong extensional flow expands the coil size in the flow direction exponentially in time, extensional flow is a sensitive "amplifier" of solvent-quality effects. It is possible, then, that extensional flow at high De, when properly interpreted, may provide a measure of solvent quality more sensitive than any other.

\section{Conclusion}

An accurate method for performing birefringence calculations using the bead-spring model is established. The calculations have been compared with both simulation results of Doyle and Shaqfeh (1998) for the bead-rod model and with the experimental measurements on a polystyrene solution. The calculations for the beadspring model agree perfectly with those for the bead-rod model, and also agree reasonably well with the experimental measurements for polystyrene solutions. Moreover, the optical-stress hysteresis during start-up followed by relaxation of extensional flow can also be predicted by the simulations and agreement is obtained with experimental measurements. The method we have developed for computing the birefringence from a beadspring model is general, and should apply to arbitrary flows just as well as it does for extensional flow.

In addition, we examined solvent-quality effects for dilute polymer solutions, by incorporating a truncated Lennard-Jones repulsion between beads to mimic excluded-volume effects in a good solvent. The expansion of the equilibrium coil size produced in a good solvent leads to stress and birefringence growth in extensional flow that is faster than in theta/poor solvents, in good agreement with experimental results. Because it expands coil dimensions in the flow direction at an exponential rate, strong extensional flow may provide a very sensitive measurement of solvent quality effects.

Acknowledgements $\mathrm{Li}$ and Larson were supported under NSF grant CTS-9707778. Li and Larson appreciate the useful communications and discussions with Dr. Tam Sridhar.

\section{References}

Andrews NC, Doufas AK, McHugh AJ (1998) Effect of solvent quality on rheological and rheooptical properties of flexible polymer solutions. Macromolecules 31:3104-3108

Batchelor GK (1970) The stress system in a suspension of force-free particles, J Fluid Mech 41:545-570.

Bird RB, Curtiss C, Amstrong RC, Hassager O (1987) Dynamics of polymeric liquids. Wiley, New York, vol II

Bossart J, Öttinger HC (1995) Orientation of polymer coils in dilute solutions undergoing shear flow: birefringence and light scattering. Macromolecules 28:5852-5860

Brandrup J, Immergut EH (1989) Polymer handbook. Wiley, New York

Cohen A (1991) A Padé approximant to the inverse Langevin function. Rheol Acta 30:270-273

Doyle PS, Shaqfeh ESG (1998) Dynamic simulation of freely-draining, flexible bead-rod chains: start-up of extensional and shear flow. J Non-Newtonian Fluid Mech 76:43-78
Doyle PS, Shaqfeh ESG, McKinley GH, Spiegelberg SH (1998) Relaxation of dilute polymer solutions following extensional flow. J Non-Newtonian Fluid Mech 76:79-110

Doyle PS, Shaqfeh ESG, Gast AP (1997) Dynamic simulation of polymers in linear flows: I. Flexible polymers. J Fluid Mech 334:251-291

Fetsko SW, Cummings PT (1995) Brownian dynamics simulations of beadspring chain models for dilute polymer 
solutions in elongational flow. $\mathrm{J}$ Rheol 39:285-299

Fuller GG (1995) Optical rheometry of complex fluids. Oxford University Press, New York

Fuller GG, Leal LG (1980) Flow birefringence of dilute polymer solutions in two-dimensional flows. Rheol Acta 19: 580-600

Grassia P, Hinch EJ (1996) Computer simulations of polymer chain relaxation via Brownian motion. J Fluid Mech 308:255-288

Hernández Cifre JG, García de la Torre J (1999) Steady-state behavior of dilute polymers in elongational flow. Dependence of critical elongational rate on chain length, hydrodynamic interaction, and excluded volume. J Rheol 43:339-358

Hinch EJ (1994) Uncoiling a polymer molecule in a strong extensional flow. J NonNewtonian Fluid Mech 54:209-230

Larson RG (1988) Constitutive equations for polymer melts and solutions. Butterworths, Boston
Larson RG (1999) The structure and rheology of complex fluids. Oxford University Press

Li L, Larson RG, Sridhar T (2000) Brownian dynamics simulations of dilute polystyrene solutions. J Rheol 44: 291-322

Li L, Larson RG (2000) Comparison of Brownian dynamics simulations with microscopic and light-scattering measurements of polymer deformation under flow. Macromolecules 33:1411-1415

Liu TW (1989) Flexible polymer chain dynamics and rheological properties in steady flows. J Chem Phys 90: $5826-5842$

Mackey JR, Das KK, Anna SL, McKinley GH (1999) A compact dual-crystal modulated birefringence-measurement system for microgravity applications. Meas Sci Technol 10:946-955

Orr NV, Sridhar T (1999) Probing the dynamics of polymer solutions in extensional flow using step strain rate experiments. J Non-Newtonian Fluid Mech 82:203-232
Peterlin A (1961) Streaming birefringence of soft linear macromolecules with finite chain length. Polymer 2:257-264

Peterlin A (1963) Mean dimensions of macromolecular coil in laminar flow. J Chem Phys 39:224-229

Rzehak R, Kromen W, Kawakatsu T, Zimmermann W (1999) Deformation of a tethered polymer in uniform flow. Euro Polym J (submitted)

Solomon MJ, Muller SJ (1996) The transient extensional behavior of polystyrene-based Boger fluids of varying solvent quality and molecular weight. J Rheol 40:837-856

Sridhar T, Nguyen DA, Fuller GG (2000) Birefringence and stress growth in uniaxial extension of polymer solutions. J Non-Newtonian Fluid Mech 90:299-315

Wiest JM (1999a) Relaxation following uniaxial extension of dilute polymer solutions. J Chem Phys 111:5205-5211

Wiest JM (1999b) Birefringence in strong flows of polymer solutions. Polymer 40: 1917-1922 